\title{
Filgrastim-sndz debuts as the first biosimilar approved in United States
}

See Commentary on page 418

I: $\mathrm{n}$ March this year, filgrastim-sndz became the first biosimilar to receive approval from the US Food and Drug Administration for use in the United States. Biosimilars are biological products that show comparable quality, efficacy, and safety to a reference drug that is already approved. In this case, filgrastim-sndz, by Sandoz, a Novartis company, is a biosimilar of Amgen's filgrastim, the reference drug or originator, which is already licensed in the United States for 5 indications, including for cancer patients undergoing treatments that deplete white blood cells, as well as those preparing for autologous peripheral blood stem-cell transplant, or those with severe, chronic neutropenia. ${ }^{1}$ The launch of the biosimilar was delayed by a lawsuit by Amgen, but it was subsequently cleared for marketing after a US Court of Appeals ruled in July that filgrastim-sndz could be marketed after September 2, 2015. The biosimilar licensure pathway is a product of the Biologics Price Competition and Innovation Act as part of an effort to encourage competition and promote lower drug prices (see sidebar, p. 421).

The filgrastim drugs are granulocyte-colony stimulating factor (G-CSF) analogs that act to boost the release of white blood cells from the bone marrow. Approval of the biosimilar was based on data from 5 pharmacokinetic and pharmacodynamic studies, 5 nonclinical studies, and 2 clinical studies, which confirmed that there were no clinically meaningful differences from the reference drug.

Overall, 20 lots of filgrastim-sndz drug product, 6 lots of EP2006 drug substance, and 10-15 lots of filgrastim were compared. The functional and physicochemical properties, product-related substances, impurities, and stability profiles were all highly similar. After treatment with a single dose of either filgrastim-sndz or filgrastim, pharmacokinetic and pharmacodynamic studies confirmed that the area under the curve and maximum concentration, the absolute neutrophil count (ANC), and CD34-positive cell counts were within $80 \%-125 \%$ of each other.

The key clinical study was the nonrandomized, doubleblind, multicenter phase 3 PIONEER study that compared the efficacy and safety of filgrastim-sndz (Zarxio) and filgrastim (Neupogen) in breast cancer patients who were treated with myelosuppressive chemotherapy. ${ }^{2}$ Eligible women aged 18 years or older, with histologically proven

\section{What's new, what's important}

Novel biological products such as monoclonal antibodies and granulocyte colony stimulating factors have revolutionized cancer treatment. However, many of the biological products are extremely expensive because of the complex nature of their production and synthesis, which means that globally millions of patients do not have access to these drugs because of their cost. In the cost-conscious and value-based environment in which we now practice in the United States, less expensive but equally effective alternatives are always welcome as a new option.

Biosimilars are biological products that show comparable quality, efficacy, and safety to a reference drug that has already been approved. Many biosimilars are in development and being approved elsewhere in the world, and the European Union and other countries are far ahead of the US in developing regulatory infrastructure and approval of biosimilars.

The Patient Protection and Affordable Care Act (Affordable Care Act) amended the Public Health Service Act to create an abbreviated licensure pathway for biological products that are demonstrated to be "biosimilar to" or "interchangeable with" a biological product that has been approved by the FDA. The first biosimilar product approved in the US filgrastim-sndz, which is a biosimilar to the US-licensed reference drug, filgrastim. The filgrastim-sndz indications are exactly same as those for filgrastim, which include chemotherapy-induced myelosuppression for patients undergoing bone marrow transplantation.

The US wholesale list price for a 300-mcg syringe of the biosimilar filgrastim-sndz is $\$ 275.66$, and for the 480 -mcg version, it is $\$ 438.98$. Filgrastim costs $\$ 324.30$ and $\$ 516.45$, respectively, for the same syringe formulations. The introduction of biosimilars as a therapy option will transform cancer treatment globally, and it is important for us to incorporate the use of FDAapproved biosimilars in our clinical practice. - Jame Abraham, MD, FACP (abrahaj5@ccf.org)

breast cancer, an Eastern Cooperative Oncology Group performance status of $\leq 2$, and adequate bone marrow function, who were scheduled to receive neoadjuvant chemotherapy with docetaxel $\left(75 \mathrm{mg} / \mathrm{m}^{2}\right)$, doxorubicin $(50 \mathrm{mg} /$ $\left.\mathrm{m}^{2}\right)$, and cyclophosphamide $\left(500 \mathrm{mg} / \mathrm{m}^{2}\right)$ were randomized equally to 1 of 4 treatment arms. Randomization was strat-

Report prepared by Jane De Lartigue, PhD. JCSO 2015;13:420-422. (2015 Frontline Medical Communications. DOI 10.12788/ jeso.0203. 


\section{Mechanism of action}

\section{G-CSF analog replenishes neutrophils}

Granulocyte-colony stimulating factor (G-CSF) is a growth factor that acts on neutrophil progenitor cells in the bone marrow by binding to its receptor on the surface of these cells. Receptor binding triggers a number of intracellular signaling pathways, including the Janus kinase and signal transducer and activator of transcription pathway.

These pathways trigger transcription of genes in the nucleus that control neutrophil proliferation, differentiation, and maturation. Ultimately, this results in the production and release of more neutrophils from the bone marrow to fight infection and boost the release of white blood cells from the bone marrow.

Filgrastim is a recombinant human G-CSF analog produced by recombinant DNA technology in Escherichia coli. As an analog of G-CSF, it exerts similar biological effects to the natural molecule. A side effect of many chemotherapeutic drugs that are used to treat cancer patients is a critical reduction in the number of white blood cells, a condition called neutropenia, therefore in patients with chemotherapy-induced neutropenia, the use of G-CSF analogs such as filgrastim can help increase the rate of recovery.

Filgrastim-sndz is a biosimilar for the US-licensed filgrastim; biosimilars are analogous to generics, but refer to biological products rather than small molecule drugs, and are not exactly the same as their reference product.

Before 2010, the only way to obtain FDA approval for a biosimilar was to go through the full new drug application process. The Biologics Price Competition and Innovation Act, signed into law in March 2010, created an abbreviated licensure pathway to accommodate biological products, enabling regulatory approval for those that could be shown to be "biosimilar" or "interchangeable" with an FDA-licensed reference product, by permitting approval without the requirement for a full complement of preclinical and clinical data. Filgrastim-sndz was the first biosimilar to be approved in the United States via this pathway.

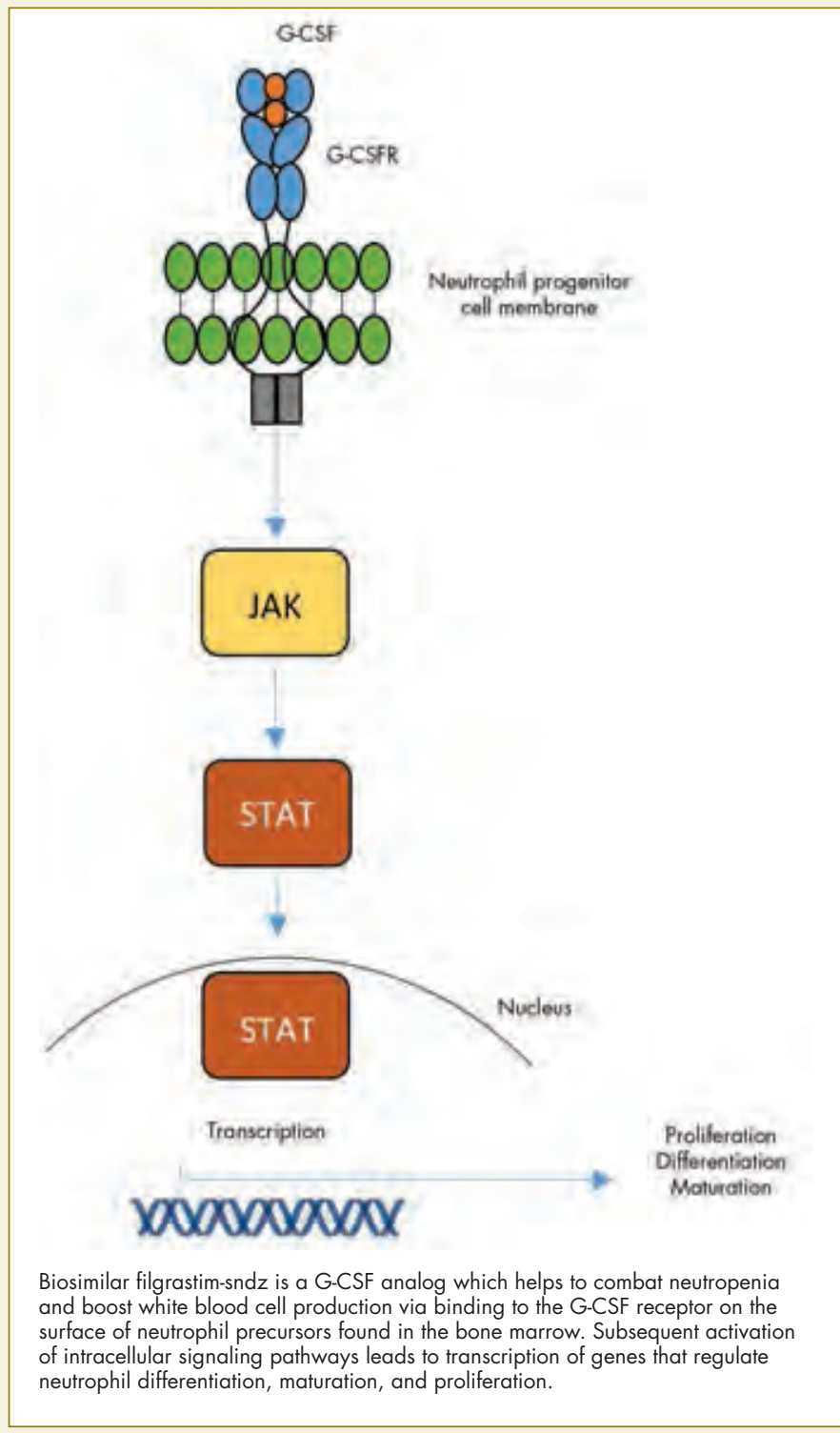

ified by adjuvant or neoadjuvant chemotherapy.

Chemotherapy was administered intravenously on Day 1 of each cycle, every 3 weeks, for 6 cycles. Patients received reference filgrastim, the biosimilar filgrastim-sndz, or 1 of 2 alternating regimens, with the filgrastim drugs administered subcutaneously at $5 \mu \mathrm{g} / \mathrm{kg}$ body weight a day from Day 2 of each cycle until ANC recovered to $10 \times 10^{9} / \mathrm{L}$ or for a maximum of 14 days.

Lab assessments were performed at screening, on Day 1 of each chemotherapy cycle, and at the end of treatment. Complete blood counts were assessed on Day 1 and then daily in Cycle 1 until ANC reached $10 \times 10^{9} / \mathrm{L}$ or until Day
15, then in cycles 2-6 on Day 1, Day 7, and daily thereafter. Body temperature was recorded on days 1-21 of each cycle and at the end of treatment. Injection site reactions were monitored after each application and concomitant medications and adverse events (AEs) were assessed at every visit. Antirecombinant human G-CSF antibody formation was examined using radioimmunoprecipitation at Day 1 of each cycle, at the end of treatment, and at study end.

The primary endpoint was duration of severe neutropenia, defined as the number of consecutive days from ANC $<0.5 \times 10^{9} / \mathrm{L}$ to $\geq 0.5 \times 10^{9} / \mathrm{L}$ during Cycle 1 of chemotherapy. Among 218 patients who were randomized, with a 
median age of 50 years, median duration since breast cancer diagnosis of 1 month, and with predominantly stage II/ III disease, the mean duration of severe neutropenia was 1.17 days for the biosimilar, compared with 1.20 days for the reference product.

Secondary endpoints included the number of patients reporting 1 or more fever episodes, total number of days of fever, incidence of febrile neutropenia (FN), and hospitalization owing to FN. In the first cycle, fever episodes occurred in $6.6 \%$ of patients in the biosimilar arm, compared with $2.8 \%$ in the reference arms, most of which resolved on the same day, with a maximum duration of 2 days in both arms. One or more episodes of FN were reported in $4.7 \%$ and $1.9 \%$ patients, respectively, and led to hospitalization in $0.9 \%$ patients in each arm.

Over all cycles, the rate of fever episodes was $15 \%$ and $4.3 \%$ for the biosimilar and reference arms, respectively, with a maximum duration of 2 days in both arms. Five percent of patients reported 1 or more episodes of $\mathrm{FN}$ in the biosimilar arm, and all of them were hospitalized, compared with no episodes in the reference arm.

Comparing alternating with nonalternating regimens, fever episodes occurred at a rate of $13.5 \%$ and $9.3 \%$, respectively, with a maximum duration of fever of 2 days, compared with 9 days. In the alternating arm, $6.7 \%$ of patients experienced 1 or more episodes of $\mathrm{FN}$, compared with $2.3 \%$ in the nonalternating arm, leading to hospitalization in $1.1 \%$ and $2.3 \%$ of patients, respectively. The depth of ANC nadir was similar across all arms, in the first cycle and subsequent cycles.

None of the patients in the study developed binding or neutralizing antibodies against G-CSF. Treatment-emergent AEs occurred in $20.6 \%$ patients in the biosimilar arm, compared with $19.6 \%$ patients in the reference arm in Cycle 1. One patient in the biosimilar arm died from pulmonary embolism, but there was no suspected relationship to the drug. Serious AEs included FN, anemia, leukopenia, diarrhea, pulmonary embolism, and hypertensive crisis. Injection site reactions were rare and mild/moderate in intensity.
Dosing of filgrastim-sndz depends on the indication. In patients with cancer who receive myelosuppressive chemotherapy, the starting dose is $5 \mu \mathrm{g} / \mathrm{kg}$ a day as a subcutaneous injection, short intravenous infusion, or continuous IV infusion; in those undergoing bone marrow transplantation, the dose is $10 \mu \mathrm{g} / \mathrm{kg}$ a day as an IV infusion for no longer than 24 hours; in patients undergoing autologous peripheral blood progenitor cell collection and therapy, the dose is $10 \mu \mathrm{g} / \mathrm{kg}$ a day as a subcutaneous injection for at least 4 days before the first leukapharesis, continued until the last leukapharesis; patients with congenital neutropenia receive a $6-\mu \mathrm{g} / \mathrm{kg}$ subcutaneous injection twice daily; and patients with cyclic/idiopathic neutropenia receive a $5-\mu \mathrm{g} /$ $\mathrm{kg}$ subcutaneous injection daily.

Filgrastim-sndz is contraindicated in patients with a history of serious allergic reactions, included anaphylaxis, to human G-CSF. The prescribing information provides further details on the risk of allergic reactions, in addition to other warnings and precautions regarding splenic rupture, acute respiratory distress syndrome, sickle cell crisis, glomerulonephritis, alveolar hemorrhage and hemoptysis, capillary leak syndrome, myelodysplastic syndrome and acute myeloid leukemia, cutaneous vasculitis, thrombocytopenia, leukocytosis, potential effects on tumor cells, and transient positive bone-imaging changes. ${ }^{3}$

References

1. US Food and Drug Administration. FDA oncologic drugs advisory committee meeting: Zarxio (filgrastim). http://www.fda.gov/downloads/AdvisoryCommittees/CommitteesMeetingMaterials/Drugs/ OncologicDrugsAdvisoryCommittee/UCM428782.pdf. Released January 7, 2015. Accessed November 8, 2015.

2. Blackwell K, Semiglazov V, Krasnozhon D, et al. Comparison of EP2006, a filgrastim biosimilar, to the reference: a phase III, randomized, double-blind clinical study in the prevention of severe neutropenia in patients with breast cancer receiving myelosuppressive chemotherapy. Ann Oncol. 2015;26:1948-1953.

3. Zarxio (filgrastim-sndz) injection, for subcutaneous or intravenous use. Prescribing information. Sandoz Inc. http://dailymed.nlm.nih. gov/dailymed/fda/fdaDrugXsl.cfm?setid=fe707775-a0ae-41b5-a74428c41889fce8\&type=display. Revised August 2015. Accessed November 8, 2015 . 